\title{
Nükak: los contactos, el Estado y la atención en salud en el norte de la Amazonía colombiana
}

\section{Kelly Peña Riveros}

(iD) https://orcid.org/0000-0001-9958-8235

Universidad Nacional General San Martín, Argentina

kelly.nukak@gmail.com

RESUMEN

Una de las razones fundamentales por las que el pueblo nükak se contactó con la sociedad mayoritaria fue la atención en salud biomédica, que con el tiempo sigue siendo una de sus vulnerabilidades primordiales. Diversas acciones judiciales durante los últimos treinta años han llamado la atención al Estado colombiano sobre la necesidad de un modelo de atención diferenciado. En este artículo indagaremos sobre el contexto histórico de dicha atención durante su proceso de contacto, con el objeto de contextualizar algunas demandas y necesidades para el análisis y ejecución de este modelo, reconociendo las diversas formas de atención que procuran los sujetos desde un enfoque relacional.

Palabras clave: nükak, atención en salud, modelo de atención, territorios nacionales, biomedicina.

\section{Nükak: contacts, the State and health care in the north of the Colombian Amazon}

ABSTRACT

One of the fundamental reasons why the Nükak people contacted the mainstream society is biomedical health care and over time it continues to be one of their primary vulnerabilities. Various legal actions 
during the last thirty years have called the attention of the Colombian State to the need for a differentiated service model. In this article we will investigate the historical context of said care during their contact process in order to contextualize some demands and needs for the analysis and implementation of this model, recognizing the various forms of care that subjects seek from a relational approach.

Keywords: Nükak, health care, model of care, National Territories, biomedicine. 


\section{INTRODUCCIÓN}

Los nükak son un pueblo nómada de cazadores-recolectores ubicados en el interfluvio del río Guaviare y el alto río Inírida en el departamento del Guaviare", noroeste de la Amazonía colombiana. Aunque la bibliografía ha indicado que su lengua pertenece a la familia lingüística makú-puinave, en recientes estudios Dany Mahecha sustenta su relación con el arawak (Agencia de Noticias UN, 2018). Los nükak están organizados en aproximadamente trece grupos locales dentro de un vasto territorio ancestral que abarca parte de las sabanas naturales de La Fuga en el Guaviare hasta un poco más allá del límite administrativo con el Guainía (Mondragón, 1992; Cabrera, Franky y Mahecha, 1999; Politis, 2007).

Cada grupo tiene su propio territorio dentro del territorio colectivo: «en la composición de los grupos locales se presenta una estructura básica, compuesta por un segmento de clan asociado con el territorio ancestral en el que habita; esta asociación permite la identificación de un grupo local con un área específica, al mismo tiempo que esta área se identifica con el grupo local» (Mahecha y Franky, 2001, p. 122). En la actualidad esas territorialidades no se usan en su totalidad, principalmente por la ocupación colona, el trabajo como recolectores en la hoja de coca comercial, sus necesidades de salud, el desplazamiento forzado y el confinamiento como producto del conflicto armado, optando por establecer campamentos /pekuji/ en sitios fijos cercanos a los caseríos colonos - algunos de ellos dentro de esta territorialidad - sin dejar de recorrer su territorio (Peña, 2021, p. 196).

El primer contacto con la sociedad mayoritaria sucedió en 1965, como profundizaremos en la primera parte, pero aparecen mencionados con anterioridad por los puinave (Marquis de Wavrin, 1948, p. 393, en Cabrera, 2002, p. 113).

El departamento del Guaviare nace con la Constitución Política de 1991. Anteriormente formaba parte de los denominados territorios nacionales en calidad de comisaría desde 1977 y para la década de 1960 pertenecía a la comisaría del Vaupés. 
Cuarenta años después de esta referencia son conocidos oficialmente en 1988 cuando un grupo de 41 personas salieron al municipio de Calamar, Guaviare (Reina, 1990, p. 18) como resultado del aumento de la colonización cocalera y, en específico, por las retaliaciones al robo de un niño colono por los nükak de la parte occidental de su territorio (Franky, 2011, p. 5). En la parte oriental, fue New Tribes Mission quien los contactó visualmente a finales de la década de 1960, y en su totalidad a principios de la de 1980, en la base llamada Laguna Pabón I. Con posterioridad se internaron más en la selva, creando la misión Laguna Pabón II, donde estuvieron hasta 1996 (Cabrera, 2015b, pp. 77-81) y al que llaman los nükak wi pairi (Peña, 2015).

Durante los primeros años pereció aproximadamente el $40 \%$ de la población por enfermedades para las que no tenían inmunidad (Mahecha y Franky, 2011, p. 10), y durante estos años fueron aumentando su presencia en los caseríos empleándose como recolectores de coca o como cocineras ${ }^{2}$. Este momento inició una serie de estudios antropológicos para la comprensión de su organización social, territorialidad, etc., que buscaban orientar la acción del Estado ante el reciente contacto.

Dadas las condiciones de vulnerabilidad inmunológica del pueblo nükak (Huertas, 2010, p. 52), su contacto y permanencia está marcado por la necesidad de atención en salud biomédica, y por ello, este artículo indaga sobre el proceso de prestación del servicio de salud biomédica en la historia de contacto y las necesidades ante la implementación de un modelo de atención diferenciado. Esta pesquisa nace de mis indagaciones durante mi estancia de trabajo en el sector salud desde 2012 hasta 2017 en los que tuve convivencia con varios grupos - especialmente entre los años 2013 a 2015-, debido a mi participación como investigadora en la propuesta de modelo de atención de actividades extralaborales. En ese período me relacioné con muchas de las personas de este pueblo, en el marco de la elaboración de mi proyecto de tesis de maestría de antropología social de la UNSAM/IDES/IDAES en Argentina.

He dividido este artículo en cuatro secciones. La primera, donde abordaré por medio de la correspondencia del Ministerio de Gobierno la salida de un segmento del pueblo, destacando la visión de los actores institucionales y su accionar frente a su aparición. En la segunda, contextualizaré el sistema de atención biomédico

2 En el Guaviare, la recolección de la hoja de coca para fines comerciales se denomina «raspar» y a los trabajadores que realizan esta labor se les denomina «raspachines». Las cocineras son las mujeres que cocinan para los trabajadores en las fincas dedicadas a la recolección de coca. 
de la comisaría del Vaupés entre las décadas de 1960 y 1980 durante el proceso de contacto de los nükak. En el tercero, indagaré sobre los procesos de atención del Estado como resultado de una sentencia judicial y el inicio de la prestación específica durante la agudización del conflicto armado hasta la actualidad. Como síntesis, finalizaré intentando analizar las formas de atención a las enfermedades entre los nükak y las necesidades de análisis dentro un modelo de atención diferenciado que reconozca sus procesos de salud/enfermedad/atención-prevención.

\section{EL CONTACTO INTERMITENTE DE 1965 Y LA PERSPECTIVA DEL ESTADO FRENTE A LOS «MACÚS»}

Aunque se registra como fecha del contacto el mes de abril de 1988, más de veinte años atrás un segmento aparece en la correspondencia del Ministerio de Gobierno. La primera comunicación sobre los nükak — para entonces macús-es del 23 de octubre de 1965, cuando el comisario del Vaupés ${ }^{3}$ escribió un telegrama al ministro de Gobierno:

Respetuosamente informo señor Ministro indígenas salvajes pertenecientes tribu macú en número aproximado cuatrocientos acosados seguramente por el hambre y la miseria están invadiendo fincas de colonos establecidos orillas río Guaviare apoderándose de comida animales domésticos etc., comisaría está tratando resolver emergencia pero considero necesidad buscar solución definitiva tratando de atraerlos vida civilizada ofreciéndoles vestuario ya que andan desnudos comida y trabajo en carretera actualmente construyese entre Calamar y San José del Guaviare infortunadamente comisaría carece medios económicos para emprender esta tarea que no admite ya espera stop apropiación para solucionar problema social a los compatriotas más necesitados de Colombia. (González, 1965, p. 36)

La autoridad local propone la integración de los nükak a la sociedad nacional por medio del uso de su fuerza de trabajo en la construcción de la carretera Calamar-San José del Guaviare, como venía sucediendo con los indígenas del complejo lingüístico Tucano Oriental que fueron trasladados hacia el río Guaviare para dicho fin (Santoyo, 2010, p. 329). En general, la orientación sobre el trabajo indígena, era la de «establecer un nuevo balance entre autoridades civiles y

3 En la década de 1960 la Comisaría del Vaupés estaba constituida por los actuales departamentos de Guainía, Guaviare y Vaupés estos últimos, elevados a la categoría de departamento con la Constitución de 1991. 
eclesiásticas en su gobierno y protección, que desembo[cara] en la flexibilización del acceso a la mano de obra» (Santoyo, 2010, p. 338).

Después, el teniente coronel de la policía Henry García informó al ministro de Gobierno que «acaba de regresar subteniente Bustamante Arboledas de sitio «charra» informando que los indios hicieron tercera incursión incendiando 3 casas de indígenas civilizados y que encuentran cerca del lugar stop colonos agrupados en 3 ranchos necesitan comidas indios salvajes están acabando con yuca y plátano y no permiten a los blancos ni indios civilizados entran a la selva conseguir animales para su sustento» ${ }^{4}($ García, 1965, p. 180)

La peligrosidad de los indígenas, dado su carácter «salvaje y famélico» — según reza otra de las comunicaciones - precisaba una respuesta urgente del gobierno nacional, razón por la cual el comisario insiste el 6 de noviembre en buscar una audiencia con el presidente de la República y el ministro de Gobierno para «tratar problema macús» (González, 1965b, p. 182). Seis días después, un marconigrama del Ministerio de Gobierno informa sobre el envío de unos mercados con maíz, arroz, panela, frijol y manteca «con destino indios macús» de La Charra (Cano, 1965, p. 189). El mismo día, la División de Asuntos Indígenas (1965) escribe al ministro con el objeto de revisar un informe para su distribución a la prensa, donde se afirma que los macús ya habían huido «temerosos del castigo de blancos y mestizos del ganado que mataron para comer» ${ }^{5}$ (p. 52) y aclaran que su capacidad institucional —ocho comisiones de asistencia y protección indígena- era incipiente para la cantidad de comunidades de todo el país, haciendo un llamado de solidaridad a otras iniciativas para «incorporarlos a la vida cristiana [y] no alimentar por breves días a aquellos compatriotas desvalidos, sino para estabilizarlos socialmente e iniciarlos en los principios de la civilización» (División de Asuntos Indígenas, 1965, p. 52).

En principio, el Ministerio se suscribe a la idea del gobierno local de que los nükak habían salido a la frontera de colonización por sus condiciones de hambre y aunque no hacen una apreciación sobre su uso como mano de obra, tampoco consideran que otras podrían ser las circunstancias que los movilizaron a tener una confrontación. En concordancia con la legislación y el pensamiento estatal del momento, plantean que solo es posible garantizar su «protección» en la medida que estos se conviertan a indígenas «civilizados».

El original se encuentra en mayúsculas.

El original se encuentra en mayúsculas. 
Casi dos meses después de estos primeros encuentros sucedió lo que algunos autores denominan «La masacre de Charras», cuando Ambrosio González —un colono cauchero que se había «fundado» ${ }^{6}$ en la hacienda llamada La Charra, a orillas del río Guaviare - entra en confrontación armada con los nükak y al parecer en presencia de la policía. De acuerdo con Cabrera (2002, p. 114), y según los nükak, este colono había talado unos huertos de chontaduro antiguos y por ello habían respondido con beligerancia. Cabrera (2002) y Cabrera, Franky y Mahecha $(1999$, p. 73$)$ describen las razones expuestas por las partes, la posibilidad de aproximadamente siete heridos y dos muertos y de la captura de una pareja de nükak que fue trasladada hasta Puerto Lleras (Meta) y que, según los nükak, no volvieron a ver. Ramón Rodríguez, un sabedor del pueblo jiw, recuerda que cuando era pequeño vio pasar a una pareja de nükak - una de ellas embarazada—, llevados por la policía en la zona que comunica La Fuga con San José del Guaviare (Rodríguez, 2020).

Ante la situación, el Ministerio de Gobierno se contactó con el comandante de la policía de los Llanos Orientales solicitando que se preste protección a los colonos y las autoridades civiles de manera pacífica y buscar contacto con los indígenas capturados para determinar las razones de las incursiones (Cano, 1965b, pp. 205-206). En esta última, el ministerio plantea por primera vez la posibilidad de consultar a los indígenas para comprender cuál era el móvil de su salida y beligerancia. Ese mismo día, el corregidor escribió al director de Territorios Nacionales ${ }^{7}$ que los indígenas detenidos llegaron al puesto de carabineros y «no han podido interpretar lengua a pesar de oírlos varios indígenas civilizados distintas tribus» (Octavio, 1965, p. 204). No se conoce si lograron comunicación con estas dos personas; sin embargo, el gobierno local insistía en la idea de insertarlos a la vida nacional como mano de obra:

colonos y caucheros de esta región ofrecen trabajo para 100 indios macús se trasladan en avión desde San José se hacen contratos con autoridades competentes stop pagáranles buenos jornales urge respuesta stop atte, Vicente

\footnotetext{
6 «Fundar» o «fundarse» es el concepto local con el que los campesinos denominan el acto de tumbar selva para establecer cultivos, viviendas y su predio en general.

7 «Se entiende por Territorios Nacionales las regiones periféricas del país que tuvieron tal nominación y un régimen especial hasta la Constitución Política de 1991, la cual, al definir una nueva estructura político-administrativa para Colombia, los convierte en departamentos» (González, 2010, p. 125). Estos territorios estaban bajo la tutela administrativa del gobierno nacional en cabeza del Ministerio de Gobierno.
} 
Londoño calle personero corregimental stop ruégole estudiar esta posibilidad e informar este despacho ${ }^{8}$ [sic]. (Suárez, 1966, p. 2).

Aunque muchas de las comunidades indígenas eran dirigidas por el Estado hacia procesos de evangelización con misioneros católicos - especialmente en el caso del Vaupés-, había una serie de pugnas centradas en el uso de la mano de obra indígena entre misioneros católicos, caucheros y funcionarios. Estos últimos, como denunció la Declaración de Miraflores de 1969, fueron cómplices de los abusos y «por tener intereses comunes y alianzas con los caucheros no cumplían sus obligaciones como funcionarios y tampoco velaban por el cumplimiento de la legislación existente sobre contratación de indígenas» (Santoyo, 2010, p. 334).

No existe otra referencia que permita precisar los impactos de este contacto esporádico en la salud de este segmento nükak, quienes corresponden al grupo local chukni a muno actualmente ubicados en las inmediaciones de Charras. Sobre su futuro, Cabrera, Mahecha y Franky (1999, p. 73) cuentan que, durante el retorno al aislamiento, este «grupo se concentró en un área que incluye las actuales veredas de Caño Seco, Charrasquera y Guanapalo», más al sur de la señalada en las apariciones.

En resumen, para el Estado, la forma de atención - aunque no se refiera específicamente a la salud - debía estar mediada por el tutelaje y la transformación del pueblo a la vida «civilizada» como mano de obra, como resultado del contexto colonial y racista. Después del retorno del segmento nükak al aislamiento, el Estado colombiano proyecta esta zona para la colonización agropecuaria, como señala en su informe Apuntes preliminares para un estudio sobre la Comisaría Especial del Vaupés de 1968:

Creemos de importancia señalar que los sectores correspondientes a la ribera derecha del Guaviare (sábanas y bosques de galería) representan para la industria agropecuaria del Vaupés una tierra prometida. En la región poco a poco comienzan a formarse haciendas ganaderas, pero en realidad no puede afirmarse que hay una industria pecuaria organizada (División de Territorios Nacionales, 1968, p. 54).

Como manifiesta la División de Territorios Nacionales (1968), el gobierno comisarial estimulaba la colonización por medio de la constitución de Juntas de Acción Comunal y la dotación de créditos, entre otros, en las «sabanas y selvas de galería que extienden desde la margen derecha de los ríos Guayabero y Guaviare,

$8 \quad$ El original se encuentra en mayúsculas. 
parte Sur de la Serranía de la Macarena, hasta el raudal de Mapiripán» (p. 66). «Esta visión de la región como "una tierra sin hombres para hombres sin tierra", ignoró abiertamente la presencia de los indígenas y negó sus derechos con el beneplácito del gobierno que promovió el avance de la colonización» (Gómez, 2018, p. 97). Muestra de ello es que el señalado informe no se menciona la presencia de los nükak, a pesar de lo acontecido tres años antes.

\section{LA ATENCIÓN EN SALUD DE LOS PUEBLOS INDÍGENAS EN LA COMISARÍA ESPECIAL DEL VAUPÉS (1960-1988)9}

Desde antes de la década de 1980, cuando se consolida una oferta de atención primaria básica en el Vaupés financiada de la cooperación holandesa, la atención biomédica de los indígenas era realizada por terceros, como los misioneros - tanto católicos como protestantes - y desde lo estatal, solo una inversión situada en los cascos urbanos y de carácter extremadamente básico. El comisario del Vaupés en 1961 afirmaba que «tienen asistencia médica las tribus más cercanas y civilizadas las cuales buscan al médico para hacerse examinar y solicitan drogas gratuitas para sus enfermedades, ya que por la pobreza en que viven, no tienen dinero con que comprarlas» (León, 1961, p. 26).

En línea con lo anterior, el Plan de desarrollo económico y social de la Comisaría del Vaupés de 1963 de la División de Territorios Nacionales afirma:

El estado general de salud en el Vaupés es el peor de toda la república. El porcentaje de tuberculosis chequeado por el Ministerio de Salud es de $85 \%$ de la población indígena. Además, en la cuenca del Vaupés, según declaraciones del director comisarial de Salud, doctor Arturo Orozco Puyo, son frecuentes los casos de edema nutricional por falta de proteínas [...] no se puede con los costos de drogas para tratamiento anti-tuberculoso, pretender curar a los enfermos. Únicamente se les alivia con una alimentación un poco mejor, para que puedan salir e ir a morir en otra parte [...] en San José del Guaviare se está construyendo un hospital que es menester terminar urgentemente; en la actualidad el servicio está atendido por un enfermero desprovisto de drogas (Guzmán y Billón, 1963, pp. 8-10).

En materia de infraestructura en toda la Comisaría solo en 1966 se termina de construir los hospitales de San José del Guaviare y Mitú (Cabrera, 2015, p. 193)

9 Establezco esta temporalidad con el objeto de reconocer el estado de la prestación de salud en el contexto de los diversos contactos que los nükak tuvieron durante este tiempo. 
y estuvieron construidos los puestos de salud de Carurú, Montfort y El Retorno (Álvarez, 1974). Estos servicios eran todavía insuficientes ya que, si comparase esta comisaría en extensión (150 $000 \mathrm{~km}^{2}$ ), sería aproximadamente Uruguay, pero sin infraestructura vial y con una incipiente y costosa navegación fluvial.

Años más tarde, en la naciente comisaría del Guaviare ${ }^{10}$, se crea el hospital del municipio de Miraflores, y a principios de la década de 1980, se amplió la infraestructura especialmente rural, como los puestos de salud de Mocuare y Araguato, por el creciente proceso de colonización cocalera y se aumenta la cantidad de promotores rurales a 38 con dotación de medicamentos y materiales educativos en salud (Convenio Colombo-Holandés, 1985, pp. 10-11). Se inician las comisiones de salud en algunas de las veredas ${ }^{11}$, con énfasis en la colonización ya que, según el convenio, la «población indígena se encontraba muy dispersa y prácticamente aislada, física, social y culturalmente» (p. 3).

Dentro de las principales causas de muerte en la población en general entre 1979 y 1984, se hallan los homicidios, las enfermedades infecciosas intestinales, otras neumopatías, el paludismo y las afecciones perinatales (Convenio ColomboHolandés, 1985, p. 78), muchas de ellas transmisibles. El Servicio de Seccional de Salud del Guaviare también tenía una débil estructura extramural, indispensable para la atención biomédica de la población indígena.

Desde los años ochenta, en el caso de los nükak de la zona oriental, la atención en salud de tipo biomédico estuvo en manos de New Tribes Mission. Con estos grupos nükak, la posibilidad de convivencia en sus campamentos se logra en octubre de 1980 y 1983 (Cabrera, 2013, p. 542) y sus primeros informes al Ministerio de Gobierno sobre el relacionamiento con los nükak datan de 1982 (Cabrera, Franky y Mahecha, 1999, p. 76).

La atención en salud biomédica es una de las razones por las cuales los nükak contactaron con los misioneros (Cabrera, 2013, p. 544). Al dialogar con Wembe, uno de los rezadores /pugnidebu/del grupo Wayari muno ${ }^{12}$, este afirma que en ese momento estaban enfrentando lo que llaman enfermedades de los blancos /kawene taayi/, para lo que no servían ni los rezos para «sacar puya» o «sacar /manap $\left./{ }^{13}{ }\right\rangle$

10 En 1977 se segrega una parte de la Comisaría del Vaupés y se crea la comisaría del Guaviare en la zona, que desde la constitución política de 1991 tiene el carácter de departamento.

11 «Vereda» alude a un tipo de subdivisión territorial dentro de un municipio, en especial en la zona rural campesina.

12 Grupo territorial que convivió con los misioneros en la zona oriental y que su territorialidad se asocia al nororiente de su territorio colectivo.

13 Dardo enviado por los espíritus o takueji. 
nilas plantas /tu'na/, porque son usadas para las enfermedades nükak/Nükak taayi/ y aunque durante la salida de los nükak a Calamar la enfermedad fue atribuida a una forma de enfermedad propia, la respuesta al padecimiento fue proporcionada por quienes fueron denominados como su causa (Kadandebe, 2017). En ese sentido, la búsqueda de los nükak de atención biomédica estaba sustentada en la valoración de las enfermedades que son producidas por los kawene $e^{14} \mathrm{y}$ las que son producidas por los nükak, tal como sugiere el mayor y que profundizaremos más adelante.

La formación de los misioneros sobre salud biomédica era básica; trataban «heridas, infecciones epidérmicas, infecciones respiratorias, quemaduras, parásitos, paludismo, vacunación, mordedura de serpiente y leishmaniasis» (Cabrera, 2007, p. 153) y afirmaban prestarla en razón a la inexistencia de otro tipo de oferta (ANTC, 1988, p. 22 en Cabrera, 2007, p. 148). Cabrera también afirma que la gripe llegaría a los segmentos nükak asociados a la misión de Laguna Pabón II en 1987, y posiblemente en estos grupos también se tuvieron muertes.

Los nükak de la zona occidental, en tanto, estaban enfrentando la llegada masiva de colonos que detonó los cultivos de coca comercial en 1978 en el Guaviare ${ }^{15}$. Así, fueron acercándose más a los colonos y en una oportunidad robaron un niño, según los nükak, porque quien se lo llevó dijo que, cuando creciera, le iba a «enseñar a trabajar como los colonos» (Franky, 2011, p. 4). Esto no solo atrajo la furia de los colonos, sino que, al parecer, «el niño estaba enfermo de gripe y los contagió, lo que ocasionó la muerte de la mayoría y solo 41 personas [...] pudieron salir ilesas trasladándose al municipio de Calamar» (Reina, 1990, p. 18). La gente de Calamar «integrados en un comité pro-indígenas $[\ldots]$ informaron $[\ldots]$ a la opinión pública indicando que los recién aparecidos indios no eran inmunes a las enfermedades de los colonos, los cual los hacía vulnerables a una epidemia» (Zambrano, 1994, p. 181). En este lugar aparecieron diferentes profesionales —entre ellos médicos y funcionarios - quienes intentaron indagar de las razones de la salida del grupo, cuando un misionero tradujo: «Los mismos nukak dicen que un "espíritu castigador" ha matado a algunos de sus hombres y el traductor interpretó que podría ser que se refirieran a la gripa o a la tuberculosis» [sic] (Wirpsa, 1988, p. 1B-3B).

En aquel momento, la División de Asuntos Indígenas se comprometió a atender su salud (Reina, 1990, p. 19), aunque la infraestructura del Servicio de Seccional de Salud del Guaviare (SSSG) creado en 1979, era bastante precario

\footnotetext{
14 Población no indígena. Aunque los nükak tienen una palabra para las personas afrodescendientes /buruna/, no diferencian cuando lo usan en plural.

15 Para profundizar sobre este asunto, ver Molano (1987).
} 
y solo tuvo una mediana ampliación hacia la zona rural en la década de 1980 . Como respuesta a la difusión de la gripe y otras enfermedades cuya letalidad se debía a la falta de inmunidad, «comenzó un periodo de intervención institucional, principalmente en la atención de salud para responder a su estadía en las zonas urbanas y, solo hasta 1993, en las áreas rurales [...] [y] empezaron a desplazarse periódicamente a San José o el Retorno para acceder a servicios de salud (Mahecha y Franky, 2011, p. 10).

\section{ATENCIÓN EN SALUD PARA LOS NÜKAK: UNA OBLIGACIÓN DE ESTADO}

En los primeros años del contacto, el fallo de la Corte Constitucional a una Acción de Tutela ${ }^{16}$ mediante sentencia T-342/1994 exigió al Ministerio de Salud estimar las medidas que dieran respuesta a la atención en salud desde el Estado colombiano, ya que, de acuerdo con la sustentación del demandante, New Tribes Mission, «gracias al manejo fluido de la lengua y al privilegio de administrar la salud, están en posibilidad de coaccionar la disposición de los indígenas frente a un mensaje ideológico que se opone a sus usos y costumbres y desarticula la cultura» (Corte Constitucional, 1994). Los misioneros empezaron a realizar el apoyo al sistema de salud desde San José del Guaviare (Franky, 2011, p. 157). De esta manera, no harían una prestación directa, sino que acompañarían $-\mathrm{y}$ todavía acompañan - canalizando pacientes, apoyando las traducciones o ayudando en su desplazamiento hacía centros de salud, entre otras carencias del sistema.

La primera acción del Estado colombiano dirigida exclusivamente a los nükak fue la realizada bajo el liderazgo del genetista Iván Yunis, mediante un convenio entre el Instituto Nacional de Salud - INS y el Servicio Seccional de Salud del Guaviare entre 1994 y 1995, con el propósito de investigar la situación de salud de los nükak y de paso realizar atención biomédica. Sin embargo, se suspendió en 1995 «ante la falta de claridad técnica, administrativa y financiera en su manejo» como producto de cambios en la coordinación técnica del proyecto por parte del INS (Cabrera, 2007, p. 82). Los años siguientes, los nükak adaptaron su movilidad y se replegaron hacia los caseríos colonos, con algunos desplazamientos hacía San José del Guaviare para ser atendidos.

16 «La acción de tutela está instituida en la Constitución Política como un mecanismo expedito que busca garantizar la protección inmediata de los derechos fundamentales» (Corte Constitucional, 2019). 
Franky, Cabrera y Mahecha (2000) documentaron la mortalidad estadísticamente con fuentes primarias y arguyen que, de los datos posteriores a 1987, «el 91,66\% corresponden a decesos causados por la "gripa", mientras el 6,41\% se ubica entre las otras causas» (Franky, Cabrera y Mahecha, 2000, p. 346). Estas causas - continúan los autores - corresponden en su orden de incidencia a prácticas chamanísticas (manap, takueyi, nemep, orojat y ebep) ${ }^{17}$, accidentes, suicidios, abortos, conflictos interétnicos, 73 sin causa y tres con causas contradictorias. Esta investigación permitió ver cuán afectado había sido el pueblo por la gripa y, de paso, dar a conocer a la sociedad nacional referencias sobre su proceso de salud/enfermedad/atención, cuestión que fue profundizada también en el documento Los Nükak: nómadas de la amazonía colombiana, además de describir las principales morbilidades en la década de 1990, las enfermedades respiratorias, las dermatológicas, el sarampión y las enfermedades diarreicas, entre otras (Cabrera, Franky y Mahecha, 1999, p. 344).

Precisamente, a inicios de 2000, la profesional Sandra Carvajal, encargada del programa de salud estatal, elaboró una propuesta que buscaba establecer un diagnóstico de la situación social de los nükak y realizar la atención en salud entre 2001 y 2003 con un componente de promoción y prevención entre los nükak y los colonos (Carvajal, 2000, pp. 28-29) ${ }^{18}$.

Con la agudización del conflicto armado desde 1997 por la incursión de las Autodefensas Unidas de Colombia —en adelante AUC — en Mapiripán ${ }^{19}$, cerca de la zona de Charras ${ }^{20}$, el grupo local chukni a muno ${ }^{21}$ se desplazó forzosamente en 2003 por la «orden de las AUC de abandonar la zona por combates» (Mahecha y Franky, 2011, p. 13). El grupo fue ubicado en el Resguardo Jiw Wuej Latj La María, cerca de San José del Guaviare, y luego desplazados al Resguardo Tucano El Refugio - sector Villa Leonor ${ }^{22}$, por peligro de inundaciones donde, desde el 9 de mayo al 24 de junio de ese año se envió a cuatro brigadas médicas.

17 En el original está escrito de acuerdo a su fonología. Varios de estos conceptos son los que denominan los nükak como «Nükak taayi» o enfermedades nükak.

18 Desde 1993 empezó a regir en Colombia la Ley 100, que crea el sistema de seguridad social integral.

19 Para profundizar sobre este suceso Corte Interamericana de Derechos Humanos. Caso de la Masacre de Mapiripán vs. Colombia. 2005. Recuperado de https://www.corteidh.or.cr/docs/ casos/articulos/seriec_134_esp.pdf

20 Este poblado es el mismo en el que sucedieron los hechos anteriormente señalados en 1965.

21 La mayor parte de los nükak que viven en inmediaciones de la vereda Charras se autodenominan así en razón a un sector de su territorio ancestral.

22 Este resguardo se encuentra al oriente del casco urbano de San José del Guaviare. 
De las 138 patologías registradas, aproximadamente el 30\% de los diagnósticos fueron infecciones respiratorias agudas no neumonía, el $24 \%$ poliparasitismo intestinal, el 7\% enfermedades diarreicas agudas y otras afecciones relacionadas con enfermedades de piel, y solo en dos casos relacionados con enfermedades urinarias y venéreas (Galindo, 2006, pp. 12-18). Durante este tiempo la atención se privilegió en los sectores aledaños a la ciudad.

En 2005, con el desplazamiento forzado de los grupos Wayari Muno de Caño Hormiga, Laguna Araguato y La Rompida, en el oriente del territorio ancestral a la vereda Agua Bonita ${ }^{23}$, se pasó de 474 atenciones ambulatorias en 2003 y 670 en 2004 a 1318 en 2005 y 1831 en 2006 (ESE Red de Servicios de Salud de Primer Nivel, 2006). La estrategia que diseña la Secretaría de Salud del Guaviare es tener promotores rurales ${ }^{24} \mathrm{en}$ los asentamientos de desplazados para el suministro de los tratamientos ordenados por los médicos y atenciones básicas, y por otro lado, realizar brigadas con médicos rurales de un día en el caso de Barrancón y dos en el caso de Agua Bonita, así como la visita de técnicos ambientales y otros profesionales de manera esporádica, entre ellas la atención psicosocial (Galindo, 2006, pp. 20-21).

En estos años es más frecuente la presencia de médicos en los asentamientos de desplazados, mientras que la atención de los grupos que se encontraban cerca al territorio ${ }^{25}$ se lleva a cabo por promotores rurales en los improvisados puestos de salud en algunas de las veredas colindantes y, de manera esporádica, se realizan consultas médicas extramurales en veredas como Caño Makú, Boquerón y Cueva Loca (Moreno, 2007).

En 2007 se amplía el tiempo de atención extramural en medicina y odontología solo a los nükak desplazados de Barrancón y Agua Bonita. Las enfermedades prevalentes son «insuficiencias respiratorias agudas, enfermedad diarreica aguda, poliparasitismo intestinal, patologías de piel como escabiosis, abscesos, piodermitis, miasis, enfermedades tropicales como paludismo, dengue, desnutrición crónica, síntomas anémicos secundarios» (Delgado, Jaimes, Díaz y Moreno,

23 Esta vereda se encuentra a quince minutos del casco urbano de San José del Guaviare. En ella se encuentra una finca de propiedad de la Alcaldía municipal.

24 La figura de promotores rurales se creó en 1956 como alternativa para la prestación de la salud en los territorios más marginados. En general eran personas de la comunidad que recibían capacitaciones en salud para atención primaria; en los últimos años la mayoría están titulados como técnicos en salud pública o auxiliares de enfermería.

25 Estos son los grupos que se instalaron cerca al río Inírida, algunos de los que se mantuvieron en el medio río Guaviare y los diversos grupos locales que habitaban las veredas Caño Makú y Guanapalo. 
2007, p. 55). A su vez, se contrata un psicólogo en un corto tiempo (junio y julio, dos veces a la semana). Sobre su salud mental, afirma:

Hay cinco tendencias que los afectan desde su propio sentir: tristeza, soledad, angustia, deseo de muerte, otros [...] también se identificaron comportamientos individuales que afectan el colectivo de la comunidad: alcoholismo, violencia con la pareja, violencia con los hijos, los hijos no hacen caso y falta de caza $[. .$.$] Y se identificaron prácticas que desde la comunidad del$ «blanco» les afectan y producen traumatismos muy serios en la comunidad y en sus patrones cotidianos de interrelación: limosna, dar alimentos, no dejar cultivar, interacción libre con los colonos, interacción de las mujeres con la FFMM, cigarrillo, cerveza, televisión, actores armados al margen de la ley (Rey, 2007, pp. 31-32)

En 2008, el equipo médico registra como morbilidad prevalente «helmintiasis, infecciones respiratorias agudas, paludismo, diarrea, infecciones de la piel, neumonía, amebiasis, bronquitis, caries dental y desnutrición» (Moreno y Galindo, 2008, p. 9). A finales de 2007, la Organización Nacional de Indígenas de Colombia contrató al médico Albeiro Riaño, quien en coordinación con la ESE de Primer Nivel atendió a las comunidades concentradas en la parte occidental, nororiental, suroriental del territorio y los asentamientos de desplazados en Agua Bonita y Barrancón. A finales de 2010, este médico presentó un diagnóstico de la morbilidad de los nükak entre 2007 y 2009 :

Están afectados sus ciclos de vida por enfermedades de tipo infecto contagioso o enfermedades «no atendidas» pero a diferencia de los Jiw, los Nukak se ven afectados de gran manera por las enfermedades transmitidas por vectores (paludismo, dengue), siendo estas de las primeras cinco causas de enfermedad por consulta externa y la primera causa de egreso hospitalario para los Nukak para los tres años evaluados [...] Igual que otras comunidades son las poblaciones de niños menores de 5 años los más afectados por la Infección Respiratoria Aguda de las Vías Respiratorias Altas, por la Enfermedad Diarreica Aguda y por el Paludismo (Riaño, 2010, p. 15).

De acuerdo con Riaño (2010), las infecciones respiratorias altas son mayores en los nükak que en la población general, además que hay una tendencia del $3,3 \%$ a convertirse en infección de las vías respiratorias bajas, como sucede con las EDA y el poliparasitismo intestinal. Sumado a esto, afirma que el paludismo y la desnutrición son una de las principales causas de hospitalización, y en el caso de la primera, de mortalidad (pp. 15-16). Finalmente, este médico hace cuatro propuestas para la atención en salud de los nükak: el fortalecimiento de 
los puestos de salud del corredor nükak en materia de dotación y personal profesional; contratación de agentes comunitarios nükak para labores de promoción y prevención; adecuación a las creencias de salud/enfermedad del pueblo, y resalta la importancia del retorno al territorio para la salud de estos (Riaño, 2010, p. 27).

Uno de los primeros acercamientos a la construcción de acuerdos y lineamientos técnicos es el Plan de Atención Integral y Diferencial para los Nükak de los asentamientos de Agua Bonita y Villa Leonor - PAID de 2009, en donde se advierte que este pueblo tiene «su propia teoría y práctica sobre el cuerpo y los procesos de salud-enfermedad» (Mahecha y Franky, 2011, p. 32). La reflexión de los nükak dentro de este plan se centró en la necesidad de micropuestos en los territorios de retorno, la capacitación de algunos nükak para ser promotores, fumigación de vectores y ellos, por su parte, se comprometieron a continuar con sus huertos y con la enseñanza de la medicina tradicional (Mahecha y Franky, 2011, p. 41).

Este mismo año surge el Auto 004 de $2009^{26}$ y se abre un ciclo de diagnósticos y planes que carecen de seguimiento, tienen una muy débil implementación (Ministerio del Interior, 2012, p. 18). Así inicia el Plan de Acción Integral para el pueblo Nükak (2011) elaborado por la CDA, Acción Social y la Unión Europea, que plantea «construir un modelo de atención en salud que respete las particularidades culturales del pueblo Nükak y permita garantizar tanto el derecho a la vida como el derecho a la cultura» (CDA, 2011, p. 104), y para ello, plantean los autores la elaboración de un estudio etnográfico sobre el cuidado del cuerpo físico y espiritual, con el fin de construir entre médicos, especialistas y los nükak el modelo y adaptarlo a cada uno de los grupos locales.

Ante la falta de avance en el Auto 004 de 2009 corroborado por la Corte, esta decidió emitir el Auto 173 de 2012, específico para los jiw y nükak, donde se ordena la elaboración e implementación del Plan Provisional Urgente de Reacción y Contingencia. Las ESE de Primer y Segundo Nivel elaboraron una propuesta conjunta en lo correspondiente a la prestación institucionalizada y extramural, con la consolidación de un equipo profesional con un equipo

26 Los Autos de seguimiento son herramientas de los magistrados de la Corte Constitucional que tienen «el fin de dar continuidad a las órdenes impartidas en la sentencia, solicitar informes y acciones concretas, hacer seguimientos puntuales, e incluso establecer indicadores de evaluación del grado de realización de los derechos» (Rodríguez Garavito y Rodríguez Franco, 2010, p. 85 en García, 2014, p. 158). El Auto 004/2009 corresponde al seguimiento a la sentencia T-025 de 2004 y conceptúa un estado de cosas inconstitucional para 33 pueblos indígenas de Colombia y ordena la elaboración de planes de salvaguardia étnica para el restablecimiento de derechos de dichos pueblos. 
psicosocial compuesto por una socióloga, una psicóloga y un traductor nükak, el fortalecimiento de la atención extramural mediante tres brigadas médicas por los corredores y de atención directa en los asentamientos, dotación de medios de transporte a los promotores de salud y contratación en cada uno de uno en cada uno de los asentamientos (Ahumada, Cárdenas, Parra y Gómez, 2012) y en cabeza de la Secretaría de Salud de San José del Guaviare, la creación dentro del Plan de Intervenciones Colectivas del componente indígena.

El ideal era realizar la atención directamente en los asentamientos nükak, así significara desplazar el equipo selva adentro. Esto fue rechazado por las FARC-EP (Fuerzas Armadas Revolucionarias de Colombia - Ejército del Pueblo), quienes informaron al equipo en un retén en inmediaciones de Caño Makú que la atención se debía hacer en los caseríos (Peña, 2015). Las atenciones realizadas en los asentamientos significaron no solo mayor atención, sino también información sobre la situación de salud de los nükak. Se descubrió, por ejemplo, en los asentamientos de desplazados, a algunos adultos mayores con triglicéridos altos y enfermedades no transmisibles (Peña, 2015).

Por otro lado, tempranamente, estos equipos comenzaron a documentar el consumo de sustancias alucinógenas no institucionalizadas, como la pasta base y marihuana en algunos niños, niñas y jóvenes de las comunidades por medio de los tamizajes realizados:

La ESE de I Nivel de San José del Guaviare viene advirtiendo desde el 2013 en los tamizajes realizados a la población Nukak [...] presentó once casos de consumo de sustancias psicoactivas y conceptúo «estos casos requieren seguimientos constantes por sus diversos factores de vulnerabilidad y una atención de rehabilitación con enfoque diferencial y etnocultural». En el 2014 [...] planteó que el 86\% de la población tamizada tiene algún tipo de consumo de sustancia psicoactiva, presentando 46 casos de consumo de SPA para la atención, la Secretaría de Salud presenta 57 casos. (Juzgado Primero Civil del Circuito Especializado en Restitución de Tierras, 2018, p. 5).

Sobre estos consumos, Sandra Pérez (2017) realizó una caracterización en la implementación del PIC Departamental. De los pocos avances durante los últimos años se encuentra la adopción de mejores sistemas de información, el aumento de la atención extramural en el área rural - aunque la presencia en general es de personal sin formación profesional y con pocas posibilidades de agencia- y se 
fortaleció medianamente el sistema de referencia y contrarreferencia ${ }^{27}$. Algunos de los procesos asociados con la atención primaria fueron construidos desde la concertación con cada uno de los grupos locales intervenidos, buscando que dichas estrategias se construyeran sobre la base de sus reflexiones y saberes; sin embargo, el formato de implementación no permite procesos, se realiza con pocos recursos y sus dinámicas no ofrecen una posibilidad real de inclusión y liderazgo de los nükak.

Antes de entrar a hablar del modelo de atención, es menester explicar al lector que, con la creación del Sistema de Seguridad Social en 1993, la atención biomédica en Colombia está en manos de empresas prestadoras de salud, en su mayoría del sector privado. La financiación del sistema no está basada en el contexto de prestación y las necesidades, sino en la oferta y la demanda, lo que ha ocasionado una crisis en territorios dispersos como el Guaviare y otros. Un ejemplo es que, en 2019, «se interpusieron 620257 acciones de tutela por presuntas violaciones a este derecho» (Defensoría del Pueblo, 2020, p. 12). Para los pueblos indígenas, resulta más desproporcionado, pues, como refiere Jimeno en Langdon (2015, p. 19), «persisten omisiones en relación con los derechos territoriales, la autonomía y las especificidades de las comunidades indígenas subsumido bajo una lógica administrativa y la racionalidad biomédica como único parámetro legítimo de la asistencia sanitaria».

\section{HACIA UN MODELO DE ATENCIÓN DIFERENCIADA EN SALUD PARA LOS NÜKAK}

A casi treinta años de prestación en salud del Estado, este se ha visto abocado a formular de un modelo de atención ante los reiterados llamados judiciales. Por eso, los primeros apartados de este artículo se dedicaron a entender cómo ha funcionado esta atención. En este plantearé algunas formas de atención como parte de un modelo de atención diferenciado, teniendo en cuenta que:

La discusión sobre lo «diferenciado» se refiere a los cimientos de una política pública marcada por una tensión permanente entre la abstracción de igualdad

27 Esto hace referencia a los procesos de traslado de pacientes desde los puestos de salud en las veredas colonas hacia el hospital en San José del Guaviare o a otras ciudades, según su grado de complejidad. Esto no implica que este tipo de referencia garantizara el traslado desde los campamentos hasta el primer nivel de atención, cuestión que muchas veces tuvieron que resolver los nükak caminando o con el apoyo de los campesinos. 
de la universalización, la estandarización de derechos regulados por el Estado y la diversidad de demandas, deseos y necesidades expresadas por individuos y grupos sociales que esperan que sus singularidades sean escuchadas y respetadas, y cuya agencia opera en el nivel concreto (Langdon y Garleno, 2017, p. 459).

Para efectos de este acápite, entendemos como modelo de atención «no solo a las actividades de tipo biomédico, sino a todas aquellas que tienen que ver con la atención de los padecimientos en términos intencionales [...]. Asumimos que [...] existen muy diferentes formas de atención a la enfermedad [...] pero además de reconocer esta diversidad» (Menéndez, 2003, p. 186). Uno de los puntos de partida es comprender qué significa la salud y enfermedad dentro de ese universal que constituye los procesos s/e/a-p (salud/enfermedad/atención-prevención). En un encuentro de rezadores nükak en diciembre de 2014, Nuyupna Yau definía salud /tüikuuchañuat/para los nükak en tres dimensiones: una en la relación con los espíritus y el cosmos, otra que tiene que ver con el vínculo entre parientes, la gente y los otros nükak, y una última de sus relaciones con yee' baka y su expresión en las plantas, el bosque o los alimentos. El cuerpo es el medio para relacionarse con estas tres dimensiones (Yau, 2014). Aunque en su exposición Nuyupna lo separa para explicarlo, en la vida cotidiana todas estas dimensiones son interdependientes y cualquier transformación en una puede afectar la otra.

Por otra parte, como ya vimos, los nükak atienden sus padecimientos en razón a la agencia nükak / no nükak. Sobre las primeras, en una conversación con Kürukü y Jarap en el asentamiento de Charras en 2014, me explicaron que dentro de las enfermedades nükak hay unas de tipo chamanístico, como el chuk'neyi o too'neyi, que es como un manto invisible que cubre a la persona y no la deja moverse hasta secarla; el manap o dardo mágico, y el ebep baka, que se parece a los temblores producidos por la epilepsia. Estas enfermedades son producto de espíritus o takueji de una persona de otro grupo que actúan sobre el cuerpo de alguien como forma de retaliación. Otros espíritus, como los nemep, pueden producir enfermedades que, en algunos casos, como describió en alguna oportunidad Cho'bobo en el hospital ante la muerte de su hija en 2014, suceden por el susto ante este espíritu y conllevan a fiebres altas y a la muerte si no se atiende a tiempo.

Este tipo de enfermedades son atendidas por un rezador /pugnidebu/que en general es el mayor de un grupo doméstico, aunque las mujeres que ya no menstrúan también podrían hacerlo. Todo esto depende de un entramado de prácticas y representaciones que forman a la persona nükak como Nükak baka' o «gente 
verdadera», concepto que, como describe Carlos Franky, «condensa un proyecto social, moral y político con el que este pueblo indígena busca reproducirse física y socialmente, fecundar y perpetuar el cosmos y guiar las relaciones con las demás gentes y seres del universo» (Franky, 2011, p. 62). A esto último cabe agregar que no se trata de una categoría anacrónica, sino que es actualizada colectivamente:

[L]a producción de gente verdadera es lo común nükak y se constituye como una biopolítica. Producir gente verdadera es ante todo una práctica en permanente actualización, que se materializa en la construcción y cuidado del cuerpo, el manejo d el medio, etc. [...] Al no ser un proceso inacabado, para los Nükak la producción de gente verdadera está en un constante riesgo que atenta contra su reproducción y contra la continuidad de este mundo. Este riesgo proviene de acciones, emociones y actitudes amorales y asociales de los mismos Nükak, de los kawene o de otros seres del cosmos, las cuales se expresan cotidianamente en situaciones como enfermedades o conflictos interpersonales. Así la vida diaria nükak se desarrolla en una tensión continua entre la actualización del proyecto de la gente verdadera y el peligro de su extinción (biológica y social) e incluso de la destrucción de este cosmos. (Franky, 2011, p. 51)

Existen una serie de estrategias nükak que «articulan dispositivos sociales como la consustancialidad, el cuidado del cuerpo y otros chamanísticos y rituales (como los ritos de paso del ciclo de vida) [que] renuevan y reproducen la formación de gente verdadera» (Franky, 2011, p. 23) y que operan en el plano de la atención-prevención. Hay otro tipo de dolencias en que los nükak acuden tanto a su conocimiento generalizado de las plantas o a la biomedicina, como las enfermedades de la piel, donde se pueden aplicar compresas de dunayi, una crema recetada por un médico, automedicada o recomendada por personal de una farmacia, o como sucede con las mordeduras de serpientes, donde usan las dos medicinas. Cuando tienen certeza de que su dolencia no depende de la agencia nükak, acuden a la biomedicina.

Un error usual es separar la medicina indígena y la biomedicina «como si ambas constituyeran sistemas culturales aislados exteriormente y homogéneos interiormente» (Ramírez, 2011, p. 51) donde hay articulaciones y principalmente conflictos en el contexto de las relaciones interétnicas «que se dan habitualmente en términos de dominación y sujeción» (Ramírez, 2011, p. 52). El contexto de prestación, cómo se pudo ver en este recorrido histórico, ha estado marcado por la marginación regional y la incapacidad del Estado de asistir con una oferta de calidad, entendiendo este último como: 
óptimo entendimiento por parte de los actores involucrados en el acto médico. Un entendimiento que implica tiempo adecuado para la consulta médica, que los servicios de salud cumplan las mínimas condiciones propias de funcionamiento resolutivo y que el personal sanitario esté en plenas condiciones para ejercer bien sus funciones (Ramírez, 2011, p. 60).

Esta necesidad ha sido expresada por los nükak en diversas oportunidades, como en septiembre de 2012, cuando un grupo de Puerto Flores se desplazó a San José del Guaviare a exigir atención y en noviembre de 2015 lo hizo un grupo de Tomachipán (Peña, 2015). También han asegurado que la presencia no es garantía de atención, porque estos funcionarios no pueden recetar medicamentos ${ }^{28}$, resolviendo enfermedades con los mínimos insumos para su atención y deben atender una cantidad importante de personas entre indígenas y no indígenas. El único asentamiento que en la actualidad cuenta con puesto de salud dentro de la comunidad está en la vereda Agua Bonita.

En el último año y medio, según informan los mükak, retiraron los promotores de salud, se hacen pocas brigadas, y se disminuyó el acceso a medicamentos ${ }^{29}$ y remisiones ${ }^{30}$. Los altos costos del traslado rural y concentración de médicos en el área urbana exigen a los nükak desplazarse buscando alternativas, como en el mes de julio de 2021 cuando los campesinos de Caño Makú tuvieron que pagar el transporte de un bebé que convulsionaba, suerte que no tuvo una mujer de Costeñita que murió esperando ser trasladada al hospital y sin la posibilidad de una atención in situ a pesar de los múltiples llamados de atención de funcionarios y misioneros (Marandúa Estéreo, 2021). A esto se suma que la institución prestadora de salud de primer nivel tiene un déficit que se aproxima a los 5000 millones de pesos (Marandúa Estéreo, 2021), así muchos de sus trabajadores llevan meses sin recibir salarios y deben poner sus vehículos para transportarse a zonas rurales inhóspitas, lejos de sus familias.

En resumen, el modelo de atención debe reconocer las formas en las que los nükak atienden sus padecimientos y las articulaciones que hay entre ellos, teniendo presente el análisis de las subjetividades en términos de hegemonía / subalternidad (Menéndez, 1994, p. 72). Este no se puede reducir a la incorporación de la medicina tradicional, pero tampoco se puede pensar sin esta, en el marco de un análisis contextualizado frente a las relaciones de poder, socioculturales y económicas que fomentan el colonialismo en el relacionamiento y las desigualdades

\footnotetext{
28 Los únicos autorizados para recetar medicamentos son los médicos tratantes.

29 El tramité era realizado por los promotores de salud.

30 Agendamiento de citas y traslado de pacientes entre los niveles de atención.
} 
socioeconómicas. Los nükak internamente discuten sobre cómo puede seguir su medicina tradicional y en ello debe aportar el modelo, pero esto no se puede hacer «sin reforzar las capacidades resolutivas de los servicios médicos y sin recursos humanos calificados [...] y sin respeto para que las medicinas tradicionales sigan avanzando en sus propios espacios» (Ramírez, 2011, p. 34).

\section{REFERENCIAS}

Ahumada, E., Cárdenas, G., Parra, H. y Gómez, R. (septiembre de 2012). Propuesta de atención en salud a la comunidad indígena Nukak Makú. E.S.E. de Primer Nivel. San José del Guaviare, Colombia.

Agencia de noticias UN (26 de octubre de 2018). Los nukak no siempre vivieron aislados. Recuperado de https://agenciadenoticias.unal.edu.co/detalle/article/ los-nukak-no-siempre-vivieron-aislados.html

ANTC (1988). Informe especial. Bogotá: División de Asuntos Indígenas.

Álvarez, J. (1974). Comisaría Especial del Vaupés. Bogotá: Ministerio de Gobierno Territorios Nacionales. Archivos oficiales - Ministerio de Gobierno. Dirección de Territorios Nacionales. Correspondencia Comisaría Especial del Vaupés, Caja 33, Carpeta 1, 41-72. Bogotá, Colombia: Archivo General de la Nación.

Cabrera, G. (2002). Los Nükak: de canibales a indígenas. Itinerario de una exclusión. Palimpsestos, 2, 144-155. Recuperado de https://revistas.unal.edu.co/index.php/ palimpsestvs/article/download/82738/72579/441664

Cabrera, G. (2007). Las Nuevas Tribus y los Indigenas de La Amazonía: Historia de una presencia protestante. Bogotá: Litocamargo.

Cabrera, G. (2013). Apuntes para una historia de los protestantes y su actuación entre los pueblos Makú del Alto río Negro - Vaupés. En P. Epps y K. Stenzel (eds.), Upper Rio Negro: cultural and linguistic interaction in Northwestern Amazonia (pp. 509-567). Río de Janeiro, Brasil: FUNAI - Museu Nacional.

Cabrera, G. (2015). Notas para una historia de una zona marginal de la Amazonía colombiana. Revista EDUCAmazônia - Educação, Sociedade e Meio Ambiente, Humaitá, XV(2), 188-215.

Cabrera, G. (2015b). Setenta años de misiones protestantes en el Vaupés, 1940-2010: el caso de la Misión Nuevas Tribus. Boletin Cultural y Bibliografico, 49(89), 67-85.

Cabrera, G., Franky, C. y Mahecha, D. (1999). Los Nükak: nómadas de la amazonía colombiana. Bogotá: Universidad Nacional. 
Cano, F. (12 de noviembre de 1965). [informe de entrega de mercados a los Macús al comisario del Vaupés]. Archivos oficiales - Ministerio de Gobierno. Dirección de Territorios Nacionales. Correspondencia Comisaría Especial del Vaupés, Caja 34, Carpeta 1, 210. Bogotá: Archivo General de la Nación.

Cano, F. (28 de diciembre de 1965b). [comunicación con el corregidor y protector de indígenas informando las medidas para el tratamiento del contacto con los Nükak]. Ministerio de Gobierno - Territorios Nacionales, Caja 32, Carpeta 1, 210. Bogotá D. C.: Archivo General de la Nación.

Carvajal, S. (2000). Proyecto integral de atención en salud y mejoramiento social y cultural de la comunidad Nukak Makú del departamento del Guaviare. Inédito, 29. San José del Guaviare: Secretaria de Salud del Guaviare.

CDA (2011). Plan de Acción Integral para el pueblo Nükak: Tuiküchañuat. San José del Guaviare: Corporación CDA.

Convenio Colombo-Holandés (octubre de 1985). Evaluación interna Guaviare. 338. Bogotá. Recuperado de https://www.ircwash.org/node/30510

Corte Constitucional. (1994). Sentencia T-342. Recuperado de https://www.corteconstitucional.gov.co/relatoria/1994/t-342-94.htm

Corte Constitucional (8 de octubre de 2019). Sentencia 462 de 2019. Recuperado de https://www.alcaldiabogota.gov.co/sisjur/listados/tematica2.jsp?subtema=32155

Corte Constitucional (2012). Auto 173. Recuperado de https://www.corteconstitucional. gov.co/relatoria/autos/2012/a173-12.htm

Defensoría del Pueblo (2020). La Tutela y los Derechos a la Salud y la Seguridad Social 2019. Bogotá D. C. Recuperado de https://www.defensoria.gov.co/public/pdf/ Estudio-La-Tutela-Derechos-Salud-Seguridad-Social-2019.pdf

Delgado, M., Jaimes, E., Díaz, I. y Moreno, D. (junio de 2007). Informe sobre el estado actual en salud de la población Nukak Makú en situación de desplazamiento ubicados en el sector de Barrancón y Agua Bonita. San José del Guaviare, Colombia: ESE Red de Servicios de Salud de Primer Nivel.

Dirección Seccional de Salud y Protección Social de Antioquía (2010). Lineamientos para la promoción de la salud con-sentido humano en Antioquia. Medellín: Universidad de Antioquia.

División de Territorios Nacionales (octubre de 1968). Apuntes preliminares para un estudio sobre la Comisaría Especial del Vaupés. Ministerio de Gobierno - División de Territorios Nacionales - Comisaría Especial del Vaupés, Caja 33, Carpeta 1, 85. Bogotá, Colombia: Archivo General de la Nación.

ESE Red de Servicios de Salud de Primer Nivel (2006). Informe Nukak Makú. San José del Guaviare, Guaviare, Colombia. 
Franky, C., Cabrera, G. y Mahecha, D. (2000). Los Nükak: Demografía, contacto y enfermedad. En A. Gómez, H. Sotomayor y A. Lesmes (eds.), Amazonía colombiana: enfermedades y epidemias. Un estudio de bioantropología histórica (pp. 319-360). Bogotá: Ministerio de Cultura.

Franky, C. (2011). «Acompañarnos contentos con la familia»: unidad, diferencia y conflicto entre los Nükak (Amazonia colombiana) (tesis de doctorado). Universidad de Wageningen. Wageningen, Países Bajos.

Galindo, C. (2006). Breve historia de los Nukak: Acciones del sector salud para los desplazados de esta etnia. 22. San José del Guaviare, Guaviare, Colombia: Secretaria de Salud del Guaviare.

García, H. (27 de octubre de 1965). Comandante de la Policía informa al Ministerio de Gobierno de la tercera incursión de los macús. Ministerio de Gobierno - Correspondencia Comisaría Especial del Vaupés, Caja 34, Carpeta 1, 210. Bogotá D. C.: Archivo General de la Nación.

García, L. (septiembre-diciembre de 2014). Los autos de seguimiento de la Corte Constitucional. ¿La Constitución de un imaginario simbólico de justicia por parte de la Corte? Análisis Político, 27(82), 149-166.

Gómez, D. (2018). Representaciones, autorrepresentaciones y negociaciones de la indianidad jiw. Bogotá: Universidad Nacional de Colombia.

González, H. (23 de octubre de 1965). Comunicación de la comisaría del Vaupés al ministro de Gobierno para informar sobre la aparición de los macús en La Charra. Ministerio de Gobierno - Correspondencia con la Comisaría del Vaupés, Caja 34, Carpeta 2, 137. Bogotá D. C.: Archivo General de la Nación.

González, H. (6 de noviembre de 1965b). Telegrama solicitando audiencia con el presidente de la república del comisario especial del Vaupés. Ministerio de Gobierno - Dirección de Territorios Nacionales, Caja 34 , Carpeta 1, 210. Bogotá: Archivo General de la Nación.

González, L. (julio-diciembre de 2010). Conocimiento y control en los confines del territorio nacional: hacia la construcción de un saber territorial, 1850-1950. Historia y Sociedad, 19, 123-142.

Guzmán, A. y Billón, F. (30 de agosto de 1963). Plan de desarrollo económico y social de la Comisaría del Vaupés. Ministerio de Gobierno - Dirección de Territorios Nacionales, Caja 33, Carpeta 1, 85. Bogotá D. C.: Archivo General de la Nación.

Huertas, B. (2010). Normatividad sobre pueblos indigenas en aislamiento, contacto reciente y contacto inicial, en la región andina. Recuperado de http://www. orasconhu.org/portal/sites/default/files/file/webfiles/doc/Normativa $\% 20 y \% 20$ propuestas $\% 20$ pueblos $\% 20$ ind $\%$ C3\%ADgenas $\% 20$ Beatriz $\% 20$ Huertas.pdf 
Juzgado Primero Civil del Circuito Especializado en Restitución de Tierras (12 de diciembre de 2018). Auto AIR-018-197. Villavicencio: Meta.

Kadandebe, W. (5 de noviembre de 2017). Entrevista sobre salud propia. K. Peña (entrevistador) y D. Niijbe, (traductor). San José del Guaviare, Colombia.

Langdon, E. y Cardoso, M. (13 de junio de 2015). Saúde Indígena: Politicas Comparadas na América Latina. Florianópolis: UFSC.

Langdon, E. y Garleno, L. (2017). Articulación entre servicios de salud y «medicina indígena»: reflexiones antropológicas sobre política y realidad en Brasil. Salud Colectiva, 13(3), 457-470. https://doi.org/10.18294/sc.2017.1117

León, E. (1961). [respuesta a la Circular Nº 068 emanada del Ministerio de Gobierno División Asuntos Indígenas]. Mitú, Vaupés: Archivo General de la Nación.

Marquis de Wavrin (1948). Les indiens sauvages de L’Améríque du sud. Vie sociale. París: Payot.

Mahecha, D. y Franky, C. (2001). Territorio y subsistencia en los Nükak. En G. Moscote, Simposio pueblos y ambientes: una mirada al pasado precolombino (Vol. Colección Memorias $\mathrm{N}^{\circ} 10$, pp. 121-130). Bogotá: Academia Colombiana de Ciencias Exactas, Físicas y Naturales.

Mahecha, D. y Franky, C. (2011). Los nükak. El último pueblo de tradición nómada contactado oficialmente en Colombia. Copenhague: IWGIA.

Marandúa Estéreo (23 de abril de 2021). \$5 mil millones sería el déficit de la ESE Primer Nivel. Recuperado de https://marandua.com.co/5-mil-millones-seria-eldeficit-de-la-ese-primer-nivel/

Marandúa Estéreo (30 de agosto de 2021). Total abandono por entes de salud a familias Nukak en Caño Mosco, Guaviare. Recuperado de https://marandua.com.co/totalabandono-por-entes-de-salud-a-familias-nukak-en-cano-mosco-guaviare/

Menéndez, E. (1994). La enfermedad y la curación. ¿Qué es medicina tradicional? Alteridades, 4(7), 71-83.

Menéndez, E. (2003). Modelos de atención de los padecimientos: de exclusiones teóricas y articulaciones prácticas. Ciência \& Saúde Coletiva, 8(1), 185-207. https://doi. org/10.1590/S1413-81232003000100014

Ministerio del Interior (noviembre de 2012). Informe de avance de la consolidación del Plan de Salvaguardia étnico del pueblo Nükak. Recuperado de https://siic.mininterior.gov.co/sites/default/files/proceso_comunitario_nukak_maku.pdf

Ministerio de Gobierno (1965). Informe Ministerio de Gobierno 6 de noviembre de 1965. En Archivos oficiales. Ministerio de Gobierno. División de Asuntos Indígenas. Correspondencia (Vol. Caja 37). Bogotá: Archivo General de la Nación. 
Ministerio de Salud y Protección Social (17-18 de enero de 2013). Acta $N^{\circ} 1$. Bogotá.

Mondragón, H. (24 de julio de 1992). La defensa del territorio Nukak. Recuperado de https://www.researchgate.net/publication/312981508_La_Defensa_del_Territorio_Nukak/citation/download

Moreno, R. (agosto de 2007). Diagnóstico de la situación actual de salud población Nukak Makú, ubicada en los sectores de Caño Makú y sus alrededores. San José del Guaviare: ESE Red de Servicios de Salud de Primer Nivel.

Moreno, R. y Galindo, C. (2008). Informe de atención en salud a población Nukak Makú en condición de desplazamiento 2003-2008. San José del Guaviare: Ese Red de Servcios de Primer Nivel y Secretaría de Salud del Guaviare.

Santoyo, A. (julio-diciembre de 2010). Disputas por el gobierno de indígenas en la antigua Comisaría del Vaupés, 1960-1968. Revista Colombiana de Antropología, Volumen 46(2), 327-352.

Octavio, M. (28 de diciembre de 1965). Oficio del corregidor encargado informando la situación a la dirección de Territorios Nacionales. Ministerio de Gobierno Territorios Nacionales, Caja 32, Carpeta 1, 210. Bogotá D. C.: Archivo General de la Nación.

Peña, K. (15 de noviembre de 2015). Diario de Campo de salud de los Nükak 20122015. San José del Guaviare.

Peña, K. (2021). Nükak: Contacto Inicial, despojo y resistencias de las mujeres en el noreste de la Amazonía colombiana. En C. Carrasco y N. Quiroga (eds.), Reexistiendo en el abya yala: desafios de la economía feminista en tiempo de pandemias (pp. 189-214). Buenos Aires, Argentina: Madreselva.

Pérez, S. (2017). Aportes a la construcción de una propuesta de promoción, prevención y atención al consumo de SPA y suicidios en los pueblos Nükak y Jiw. San José del Guaviare: Secretaría de Salud del Guaviare.

Politis, G. (2007). Nukak: Crónicas del contacto. En Parellada, A. Pueblos indigenas en aislamiento voluntario y contacto inicial en la Amazonía y el Gran Chaco. Copenhague: IGWIA.

Ramírez, S. (2011). Salud intercultural: Crítica y problematización a partir del contexto boliviano. La Paz: ISEAT.

Reina, L. (10 de marzo de 1990). Actividades relacionadas con los Nükak. Mopa Mopa, $1(5), 17-28$.

Rey, N. (octubre de 2007). Atención psicosocial a desplazados comunidad Nukak Makú. 41. San José del Guaviare, Guaviare, Colombia: ESE Red de Servicios de Salud de Primer Nivel. 
Riaño, A. (2010). Diagnóstico provisional de la situación del pueblo indígena Nukak Makú. 30. San José del Guaviare, Guaviare, Colombia: Organización Nacional de Indígenas de Colombia.

Rodríguez, R. (28 de octubre de 2020). Entrevista sobre la situación de la mujer Jiw. (K. Peña, entrevistador)

Suárez, F. (3 de enero de 1966). Comunicación solicitando traslado de indígenas macús para trabajar con colonos y caucheros. Ministerio de Gobierno - Dirección de Territorios Nacionales - Correspondencia Comisaría Especial del Vaupés, Caja 34, Carpeta 2, 285. Mitú, Vaupés, Colombia: Archivo General de la Nación.

Valencia, J. (6 de noviembre de 1965). Informe sobre la visita realizada a la Comisaría especial del Vaupés, San José del Guaviare, Mitú y Momfort a solicitud del INCORA. Ministerio de Gobierno - Correspondencia con la Comisaría del Vaupés, Caja 34, Carpeta 2, folios 7-137. Bogotá D. C.

Wirpsa, L. (22 de abril de 1988). Un espíritu castigador persigue a los Nükak. El Espectador.

Yau, N. (18 de diciembre de 2014). Diálogo sobre el concepto de salud - Encuentro de Pugnidebu - I fase de la construcción del SISPI. (K. Peña, entrevistador, y C. Yau, traductor).

Zambrano, C. (enero-diciembre de 1994). El contacto con los Nükak del Guaviare. Revista Colombiana de Antropología, XXXI, 179-193. 